\title{
Salidroside induces apoptosis in human ovarian cancer SKOV3 and A2780 cells through the p53 signaling pathway
}

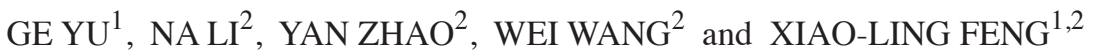 \\ ${ }^{1}$ Department of Gynecology of Traditional Chinese Medicine, Heilongjiang University of Chinese Medicine; \\ ${ }^{2}$ Department of Gynecology of Traditional Chinese Medicine, The First Affiliated Hospital of \\ Heilongjiang University of Chinese Medicine, Harbin, Heilongjiang 150001, P.R. China
}

Received August 6, 2017; Accepted November 16, 2017

DOI: $10.3892 / \mathrm{ol} .2018 .8090$

\begin{abstract}
Salidroside is one of the most potent compounds extracted from the plant Rhodiola rosea, and its cardiovascular protective effects have been studied extensively. However, the role of salidroside in human ovarian carcinoma remains unknown. The aim of the current study was to investigate the effects of salidroside on the proliferation and apoptosis of SKOV3 and A2780 cells using MTT assay and acridine orange/ethidium bromide staining. Salidroside activated caspase-3 and upregulated the levels of apoptosis-inducing factor, Bcl-2-associated X and Bcl-2-associated death promoter (Bad) proteins. Furthermore, salidroside downregulated the levels of Bcl-2, p-Bad and X-linked inhibitor of apoptosis proteins. Salidroside activated the caspase-dependent pathway in SKOV3 and A2780 cells, upregulating p53, p21 ${ }^{\text {Cip1/Waf1 }}$ and $\mathrm{p} 16^{\text {INK4a }}$. These results suggest that the $\mathrm{p} 53 / \mathrm{p} 21^{\text {Cip1/Waf1 }} / \mathrm{p} 16^{\text {INK4a }}$ pathway may serve a key function in salidroside-mediated effects on SKOV3 and A2780 cells. The current findings indicate that salidroside may be a promising novel drug candidate for ovarian cancer therapy.
\end{abstract}

\section{Introduction}

Ovarian cancer (OC) is the most lethal gynecological malignancy (1). For epithelial OC (EOC), the prognosis for premenopausal women with early-stage EOC is favorable (2). In the past few decades, patients with EOC were treated with the latest chemotherapeutic drugs and surgical techniques, but the 5-year survival rate was still $\leq 40 \%$ (3). A previous study reported that EOC demonstrates genomic instability (4). During treatment, numerous patients with EOC have recurrence and

Correspondence to: Dr Xiao-Ling Feng, Department of Gynecology of Traditional Chinese Medicine, The First Affiliated Hospital of Heilongjiang University of Chinese Medicine, 24 He Ping Road, Harbin, Heilongjiang 150001, P.R. China

E-mail: doctorfxl@163.com

Key words: salidroside, apoptosis, p53 signaling pathway, ovarian cancer become resistant to chemotherapy, indicating that new treatment strategies are required $(5,6)$. Therefore, numerous studies have been performed to identify effective therapeutic agents and their associated mechanisms of action $(6,7)$.

Salidroside, a p-hydroxyphenethyl- $\beta$-D-glucoside (or phenylpropanoid glycoside), is one of the major active ingredients extracted from Rhodiola rosea and has a long history of use in Chinese medicine (8-11). Salidroside has primarily been used as a brain tonic, a roborant or headache relief agent $(8,12)$. Recently, salidroside has been studied in experimental animals for its protective effects against hypoxia, cold, radiation and heavy physical exercise (11). It has been demonstrated that salidroside has various pharmacological properties (13), including antiaging (14), anticancer (15), anti-inflammation, hepatoprotective and antioxidative effects (16).

The aim of the current study was to investigate the effects of salidroside on OC, and to determine whether it may be a new therapeutic candidate in the treatment of OC.

\section{Materials and methods}

Cell culture. Human ovarian cancer cell lines, SKOV3 and A2780 from the American Type Culture Collection (Manassas, VA, USA), were cultured in RPMI 1640 medium (Hyclone; GE Healthcare Life Sciences, Logan, UT, USA) containing $10 \%(\mathrm{v} / \mathrm{v})$ heat-inactivated fetal bovine serum (Invitrogen; Thermo Fisher Scientific, Inc., Waltham, MA, USA) at $37^{\circ} \mathrm{C}$ in an incubator containing humidified air with $5 \%(\mathrm{v} / \mathrm{v}) \mathrm{CO}_{2}$.

Cell viability assay. SKOV3 and A2780 cells were seeded in 96 -well plates at $5 \times 10^{3}$ cells/well and treated with salidroside at different concentrations $(0,50,100,500,1,000$ or $2,000 \mu \mathrm{mol} / \mathrm{l}$ ) for $48 \mathrm{~h}$, at $37^{\circ} \mathrm{C}$ in an incubator containing humidified air with $5 \%(\mathrm{v} / \mathrm{v}) \mathrm{CO}_{2}$. A total of $10 \mu \mathrm{l} \mathrm{MTT}$ $(5 \mathrm{mg} / \mathrm{ml})$ was added to each well and incubated in the dark at $37^{\circ} \mathrm{C}$ for $4 \mathrm{~h}$. The supernatant was removed and replaced with $150 \mu$ l dimethyl sulfoxide. The plates were oscillated for $10 \mathrm{~min}$ and the absorbance was measured at $490 \mathrm{~nm}$.

Acridine orangelethidium bromide (AO/EB) staining. SKOV3 and A2780 cells in the logarithmic growth phase were deposited in $2 \times 10^{3}$ cells each well of a 96-well plate. 
A

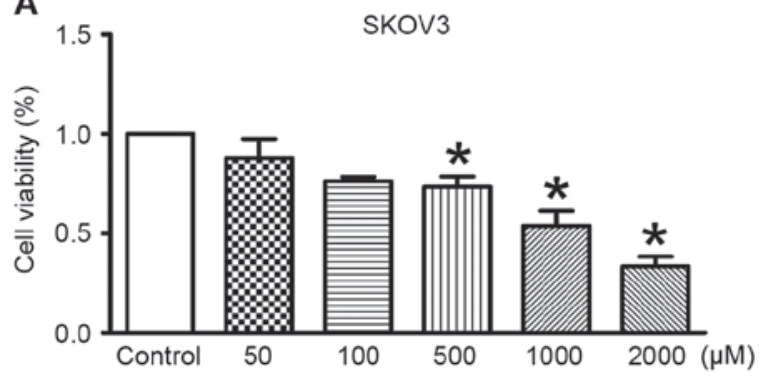

B

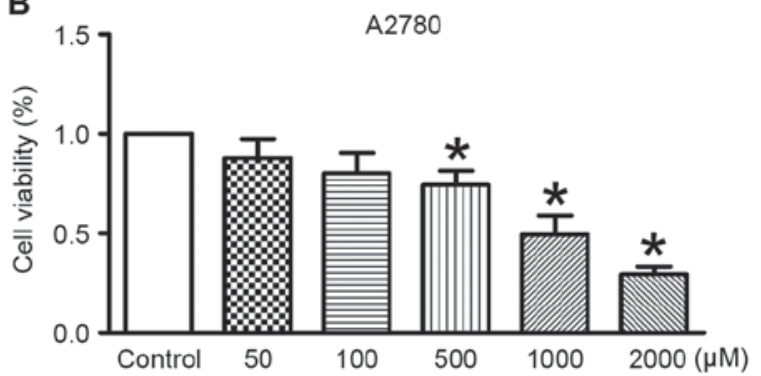

Figure 1. Salidroside inhibits the viability of SKOV3 and A2780 cells. (A) SKOV3 and (B) A2780 cells were treated with salidroside. Relative cell viability was determined by MTT assay. ${ }^{*} \mathrm{P}<0.05$ vs. control; $\mathrm{n}=6$ independent experiments for each condition.

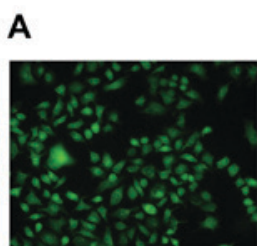

Control

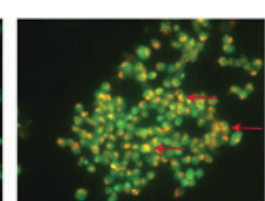

Salidroside

SKOV3

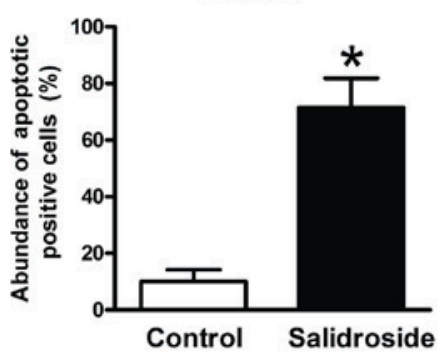

B



Control

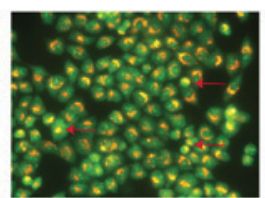

Salidroside
A2780



Figure 2. Salidroside induces apoptosis in SKOV3 and A2780 cells. Acridine orange/ethidium bromide staining was performed to detect changes in the nuclei of (A) SKOV3 and (B) A2780 cells. Red arrows indicate apoptotic cells. Magnification, x200. Data are expressed as the mean \pm standard error of the mean; $\mathrm{n}=3$ for each group. ${ }^{*} \mathrm{P}<0.05$ vs. control.

After $24 \mathrm{~h}$, cells were subsequently treated with salidroside $(0$ or $1,000 \mu \mathrm{mol} / 1)$ for $48 \mathrm{~h}$ at $37^{\circ} \mathrm{C}$ in an incubator containing humidified air with $5 \%(\mathrm{v} / \mathrm{v}) \mathrm{CO}_{2}$, stained with $\mathrm{AO}(100 \mu \mathrm{g} / \mathrm{ml}$ in PBS) and $\mathrm{EB}(100 \mu \mathrm{g} / \mathrm{ml}$ in PBS $)$ at room temperature and analyzed by fluorescence microscopy at x200 magnification (17). Five fields were randomly selected for study. Cell apoptosis (\%) was defined as the number of apoptotic cells divided by the total number of cells $(18,19)$.

Antibodies and western blotting. SKOV3 and A2780 cells [treated with salidroside $(1,000 \mu \mathrm{mol} / 1$; salidroside group) or PBS (control group) for $48 \mathrm{~h}$ at $37^{\circ} \mathrm{C}$ in an incubator containing humidified air with $5 \%(\mathrm{v} / \mathrm{v}) \mathrm{CO}_{2}$ ] were collected and lysed in ice-cold radioimmunoprecipitation assay buffer (Roche Diagnostics, Basel, Switzerland). The protein concentration of the lysates was measured using a bicinchoninic acid Protein Assay kit (Pierce; Thermo Fisher Scientific, Inc.) according to the protocol of the manufacturer. Cell lysates were separated by $10 \%$ SDS-polyacrylamide gel electrophoresis and electrotransferred to nitrocellulose membranes (Pall Corporation, Port Washington, NY, USA). The membranes were blocked using 5\% skim milk then incubated with primary antibodies against $\beta$-actin (cat. no. 3700; 1:2,000 dilution; Cell Signaling Technology, Inc.,
Danvers, MA, USA), p53 (cat. no. 2524; 1:500 dilution; Cell Signaling Technology, Inc.), p21 Cip1/Waf1 (cat. no. 610233; 1:500 dilution; BD Biosciences, Franklin Lakes, NJ, USA), p16 ${ }^{\text {INK4a }}$ (cat. no. sc-53392; Santa Cruz Biotechnology, Santa Cruz, CA, USA), Bcl-2-associated X protein (Bax) (cat. no. 5020; 1:1,000 dilution; Cell Signaling Technology, Inc.), Bcl-2-associated death promoter (Bad) (cat. no. 9268; 1:500 dilution; Cell Signaling Technology, Inc.), and p-Bad (cat. no. 9291; 1:500 dilution; Cell Signaling Technology, Inc.), Bcl-2 (cat. no. 15071; 1:1,000 dilution; Cell Signaling Technology, Inc.), apoptosis-inducing factor (AIF) (cat. no. 5318; 1:1,000 dilution; Cell Signaling Technology, Inc.) and X-linked inhibitor of apoptosis (XIAP) (cat. no. 14334; 1:1,000 dilution; Cell Signaling Technology, Inc.) at $4^{\circ} \mathrm{C}$ overnight. The membranes were incubated with rabbit (cat. no. 7054; 1:5,000 dilution; Cell Signaling Technology, Inc.) or mouse (cat. no. 7056; 1:5,000 dilution; Cell Signaling Technology, Inc.) secondary antibodies at room temperature for $1.5 \mathrm{~h}$. Immunoreactive bands were visualized using an enhanced chemiluminescence reagent (GE Healthcare, Chicago, IL, USA). Intensities of immunoreactive bands were determined by densitometric analysis using ImageJ software (version 1.61; National Institutes of Health, Bethesda, MD, USA) and normalized against $\beta$-actin. 
A
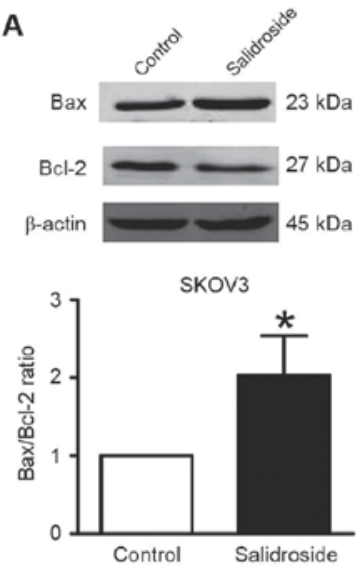

B
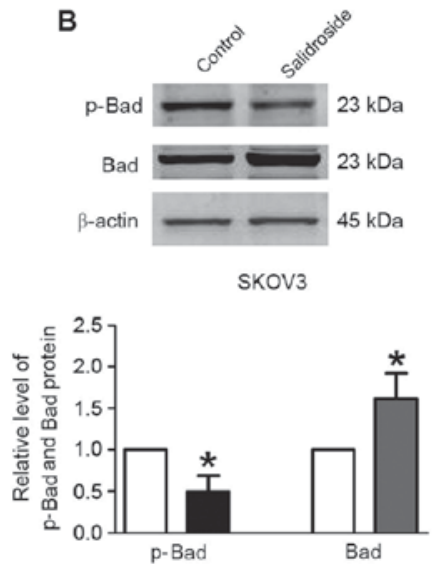

C



D
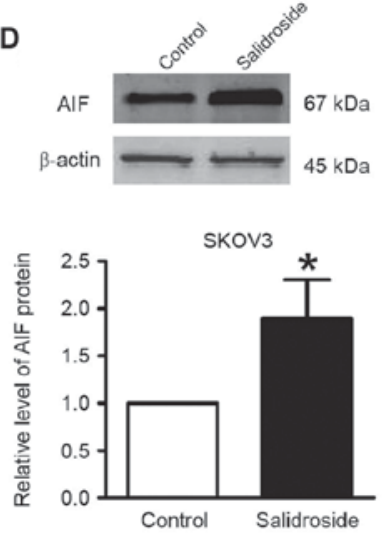

E

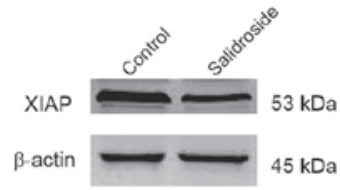

SKOV3

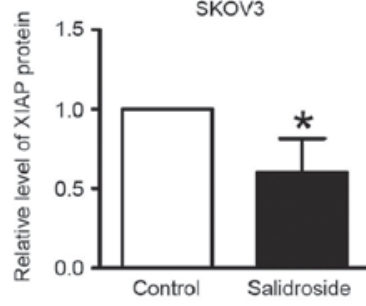

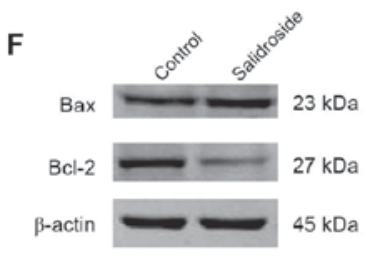

A 2780

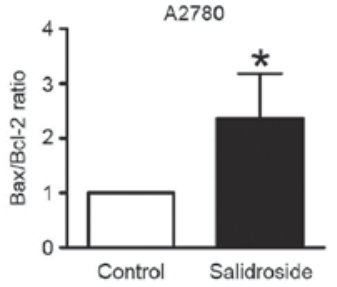

G

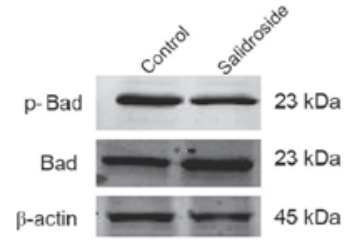

A 2780
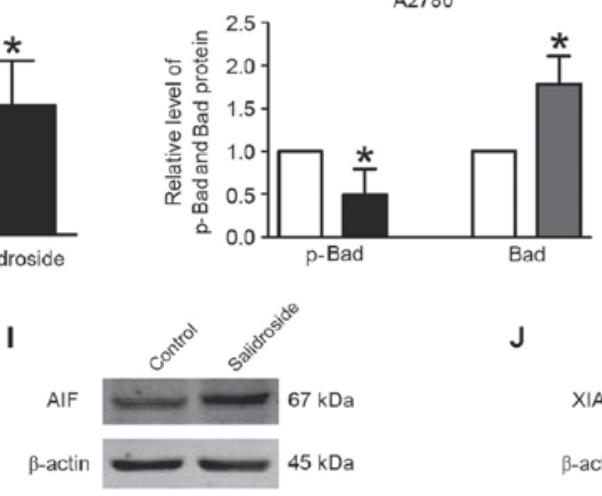

H

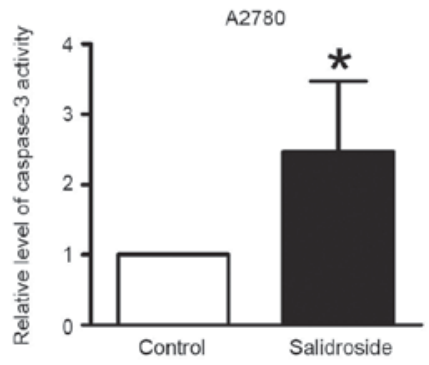

\section{J}
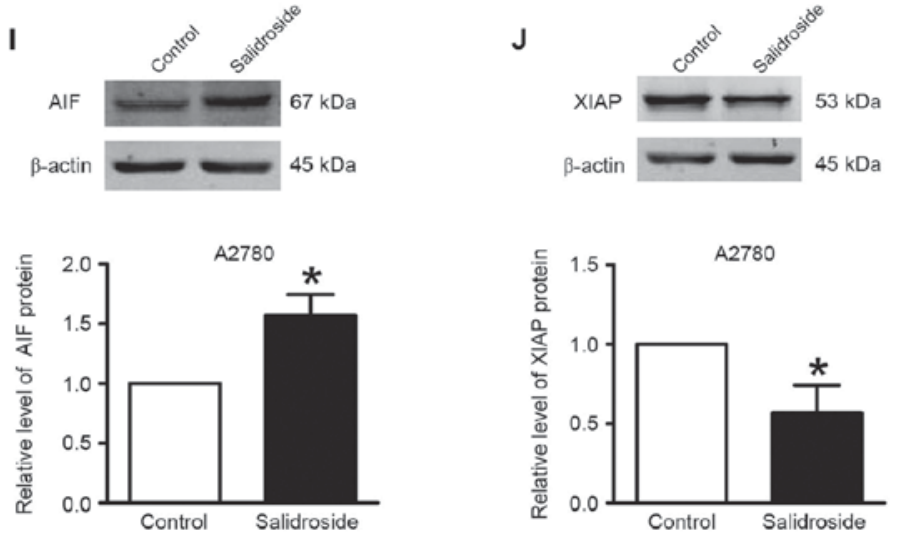

Figure 3. Salidroside alters p-Bad, Bad, Bax, Bcl-2, AIF and XIAP expression, and promotes caspase-3 activation. Western blotting was used to detect protein expression. Relative protein expression was normalized to $\beta$-actin. Caspase-3 activity was measured using a commercial assay kit. (A) Bax and Bcl-2 expression, (B) Bad and p-Bad expression, (C) caspase-3 activity, (D) AIF expression and (E) XIAP expression in SKOV3 cells treated with salidroside. (F) Bax and Bcl-2 expression, (G) Bad and p-Bad expression, (H) caspase-3 activity, (I) AIF expression and (J) XIAP expression in A2780 cells treated with salidroside. Data are expressed as the mean \pm standard error of the mean; $n=3$ independent experiments for each group. " $\mathrm{P}<0.05$ vs. control. Bax, Bcl-2-associated $\mathrm{X}$ protein; Bad, Bcl-2-associated death promoter; AIF, apoptosis-inducing factor; XIAP, X-linked inhibitor of apoptosis. 


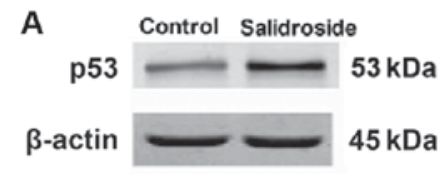

SKov3




SKov3


A2780

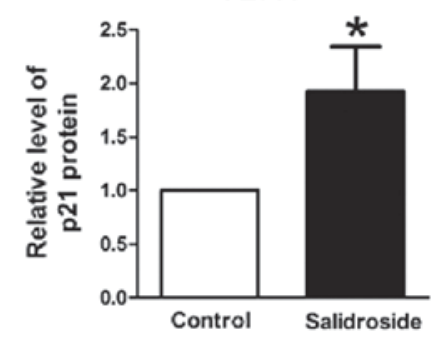



SKOV3


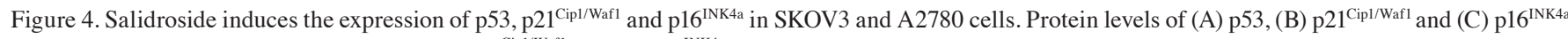
in SKOV3 cells. Protein levels of (D) p53, (E) p21 $1^{\mathrm{Cip} / \mathrm{Wafl}}$ and (F) p16 $6^{\mathrm{INK4a}}$ in A2780 cells. Data are expressed as the mean \pm standard error of the mean; $\mathrm{n}=3$ for each group. "P<0.05 vs. control.

Caspase-3 activity assay. A caspase-3 activity assay kit (cat no. C1115; Beyotime Institute of Biotechnology, Haimen, China) was used to test the activity of caspase- 3 following treatment with salidroside ( 0 or $1,000 \mu \mathrm{mol} / \mathrm{l})$ for $48 \mathrm{~h}$ in ovarian cancer (SKOV3 and A2780) cells. Caspase activity was expressed as a percentage of the control.

Statistical analysis. Data are presented as the mean \pm standard error of the mean of three independent experiments. Statistical analysis was conducted using SPSS 19.0 software (IBM Corp., Armonk, NY, USA) and illustrated using GraphPad Prism 5.0 (GraphPad Software, Inc., La Jolla, CA, USA). Statistical significance was determined using Student's t-test to compare two groups or analysis of variance with Tukey's post hoc test to compare multiple groups. $\mathrm{P}<0.05$ was considered to indicate a statistically significant difference.

\section{Results}

Assessment of OC cell viability after treatment with salidroside. Across different concentrations (500, 1,000 or $2,000 \mu \mathrm{mol} / \mathrm{l}$ ) of salidroside treatment, cell viability was significantly inhibited compared with the control (Fig. 1). The data revealed that salidroside treatment at $1,000 \mu \mathrm{mol} / 1$ for $48 \mathrm{~h}$ inhibited viability of SKOV3 and A2780 cells by $~ 50 \%$. For this reason, in subsequent experiments $1,000 \mu \mathrm{mol} / 1$ was used as salidroside treatment.
Salidroside induces apoptosis in SKOV3 and A2780 cells. Cell viability is a balance between cell proliferation and apoptosis (20). AO/EB staining revealed that salidroside significantly increased the rate of apoptosis in SKOV3 (Fig. 2A) and A2780 cells (Fig. 2B).

Salidroside activates pro-apoptotic signaling pathways. To explore the functional mechanisms of salidroside, the expression of Bax, Bcl-2, Bad, p-Bad, AIF and XIAP proteins was investigated by western blotting in SKOV3 (Fig. 3A-E) and A2780 (Fig. 3F-J) cells. The results indicated that treatment with salidroside significantly upregulated the ratio of $\mathrm{Bax} / \mathrm{Bcl}-2$ and the expression of Bad and AIF, but significantly downregulated p-Bad and XIAP expression in SKOV3 and A2789 cells. Meanwhile, caspase-3 activity was significantly increased by salidroside in SKOV3 (2.1-fold increase) and A2780 (2.5-fold increase) cells.

Salidroside activates p53 signaling pathways. To define whether p53 signaling was involved in salidroside-induced apoptosis, the protein levels of p53, p21 $1^{\text {Cip1/Waf1 }}$ and p16 ${ }^{\text {INK4a }}$ were evaluated. They were identified to be significantly upregulated in SKOV3 (Fig. 4A-C) and A2780 (Fig. 4D-F) cells after treatment with salidroside compared with the control. These results

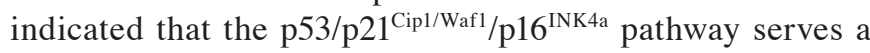
critical function in salidroside-induced apoptosis in SKOV3 and A2780 cells. 


\section{Discussion}

In the present study, in vitro experiments demonstrated that salidroside exerts potent anti-proliferative effects on SKOV3 and A2780 cells by inducing apoptosis. Furthermore, the mechanisms underlying the anticancer effects of salidroside on $\mathrm{OC}$ were investigated. The results indicated that activation of the caspase-3-dependent pathway and the p53 signaling pathway were involved in mediating these salidroside-induced effects. Therefore, the present results may provide an experimental basis for the potential role of salidroside in treating OC.

The current results are consistent with previous reports demonstrating that salidroside exerts anticancer effects in breast carcinoma $(15)$, human fibrosarcoma $(21,22)$ and neuroblastoma (23). The mechanism of action of salidroside has been reported to involve autophagy (24). However, the effect of salidroside in OC is not yet fully understood.

The present data suggested that salidroside has antiproliferative and pro-apoptotic effects on OC cells. It was revealed that salidroside inhibited the viability of SKOV3 and A2780 cells. Furthermore, AO/EB staining indicated that salidroside induced apoptosis in OC cells.

The effect of salidroside on the regulation of gene expression has been studied previously. Numerous studies have revealed that $\mathrm{Bax}, \mathrm{Bcl}-2$ and caspase- 3 are involved in apoptosis in SKOV3 and A2780 cells (25). Liu et al (26) identified that caspase-mediated cleavage of Beclin1 inhibits autophagy and promotes apoptosis in SKOV3 cells. Furthermore, andrographolide radiosensitizes human SKOV3 cells (27), and miRNA-149 modulates the chemosensitivity of A2780 cells by modulating Bax protein expression (28). In the present study, it was demonstrated that salidroside could induce Bcl-2 and Bax expression, and upregulate caspase-3 in SKOV3 and A2780 cells. In addition, the ratio of $\mathrm{Bcl}-2 / \mathrm{Bax}$ was /decreased, indicating that salidroside promotes apoptosis in OC. Bad is known to regulate apoptosis by forming heterodimers with Bax and Bcl-2 (29). XIAP inhibits activation of caspases by binding to them, preventing apoptosis of tumor cells (30). In the present study, Bad protein was significantly increased while p-Bad and XIAP levels were significantly decreased following treatment with salidroside. These results demonstrate that salidroside could induce apoptosis via caspase-3-dependent apoptosis signaling in OC $(31,32)$. Furthermore, p53 can influence apoptosis by regulating Bcl-2 (33). Previous studies have revealed that $>50 \%$ of tumors are associated with p53 gene mutation; wild-type p53 gene therapy has been suggested to strengthen sensitivity to cisplatin in SKOV3 cells (34-36). In the present study, it was demonstrated that salidroside has the ability to induce OC apoptosis. Salidroside also promoted the expression of p53, p21 ${ }^{\text {Cip1/Waf1 }}$ and p16 ${ }^{\text {INK4a }}$ expression in SKOV3 and A2780 cells. It was identified that salidroside promotes the expression of caspase- 3 and activation of the

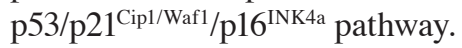

In summary, salidroside was demonstrated to reduce cell viability and promote apoptosis in OC. Furthermore, it was identified that salidroside activates caspase- 3 and the

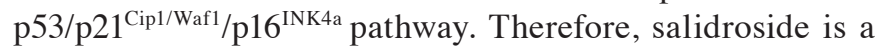
promising new approach for the treatment of OC, but its underlying mechanism needs to be explored further.

\section{Competing interests}

The authors declare that they have no competing interests.

\section{References}

1. Siegel R, Ma J, Zou Z and Jemal A: Cancer statistics, 2014. CA Cancer J Clin 64: 9-29, 2014.

2. Vang R, Shih IeM and Kurman RJ: Ovarian low-grade and high-grade serous carcinoma: Pathogenesis, clinicopathologic and molecular biologic features, and diagnostic problems. Adv Anat Pathol 16: 267-282, 2009.

3. Vaughan S, Coward JI, Bast RC Jr, Berchuck A, Berek JS, Brenton JD, Coukos G, Crum CC, Drapkin R, Etemadmoghadam D, et al: Rethinking ovarian cancer: Recommendations for improving outcomes. Nat Rev Cancer 11: 719-725, 2011.

4. Landen CN Jr, Birrer MJ and Sood AK: Early events in the pathogenesis of epithelial ovarian cancer. J Clin Oncol 26: 995-1005, 2008.

5. Friedlander M, Rau J, Lee CK, Meier W, Lesoin A, Kim JW, Poveda A, Buck M, Scambia G, Shimada M, et al: Quality of life in patients with advanced epithelial ovarian cancer (EOC) randomized to maintenance pazopanib or placebo after first-line chemotherapy in the AGO-OVAR 16 trial. Measuring what matters-patient centered endpoints in trials of maintenance therapy. Ann Oncol Dec 18, 2017. Doi: 10.1093/annonc/mdx796.

6. Paik ES, Kim JH, Kim TJ, Lee JW, Kim BG, Bae DS and Choi CH: Prognostic significance of normal-sized ovary in advanced serous epithelial ovarian cancer. J Gynecol Oncol 29: e13, 2018.

7. Westin SN, Herzog TJ and Coleman RL: Investigational agents in development for the treatment of ovarian cancer. Invest New Drugs 31: 213-229, 2013.

8. Jin N, Wu H, Miao Z, Huang Y, Hu Y, Bi X, Wu D, Qian K, Wang L, Wang C, et al: Network-based survival-associated module biomarker and its crosstalk with cell death genes in ovarian cancer. Sci Rep 5: 11566, 2015.

9. Darbinyan V, Kteyan A, Panossian A, Gabrielian E, Wikman G and Wagner $\mathrm{H}$ : Rhodiola rosea in stress induced fatigue-a double blind cross-over study of a standardized extract SHR-5 with a repeated low-dose regimen on the mental performance of healthy physicians during night duty. Phytomedicine 7: 365-371, 2000.

10. Spasov AA, Wikman GK, Mandrikov VB, Mironova IA and Neumoin VV: A double-blind, placebo-controlled pilot study of the stimulating and adaptogenic effect of Rhodiola rosea SHR-5 extract on the fatigue of students caused by stress during an examination period with a repeated low-dose regimen. Phytomedicine 7: 85-89, 2000.

11. Panossian A, Wikman G and Sarris J: Rosenroot (Rhodiola rosea): Traditional use, chemical composition, pharmacology and clinical efficacy. Phytomedicine 17: 481-493, 2010.

12. Mao GX, Xing WM, Wen XL, Jia BB, Yang ZX, Wang YZ, Jin XQ, Wang GF and Yan J: Salidroside protects against premature senescence induced by ultraviolet $\mathrm{B}$ irradiation in human dermal fibroblasts. Int J Cosmet Sci 37: 321-328, 2015.

13. Barhwal K, Das SK, Kumar A, Hota SK and Srivastava RB: Insulin receptor A and Sirtuin 1 synergistically improve learning and spatial memory following chronic salidroside treatment during hypoxia. J Neurochem 135: 332-346, 2015.

14. Huang X, Zou L, Yu X, Chen M, Guo R, Cai H, Yao D, $\mathrm{Xu} \mathrm{X}$, Chen Y, Ding C, et al: Salidroside attenuates chronic hypoxia-induced pulmonary hypertension via adenosine A2a receptor related mitochondria-dependent apoptosis pathway. J Mol Cell Cardiol 82: 153-166, 2015.

15. Lai MC, Lin JG, Pai PY, Lai MH, Lin YM, Yeh YL, Cheng SM, Liu YF, Huang CY and Lee SD: Protective effect of salidroside on cardiac apoptosis in mice with chronic intermittent hypoxia. Int J Cardiol 174: 565-573, 2014.

16. Zhao G, Shi A, Fan Z and Du Y: Salidroside inhibits the growth of human breast cancer in vitro and in vivo. Oncol Rep 33: 2553-2560, 2015.

17. Yang ZR, Wang HF, Zuo TC, Guan LL and Dai N: Salidroside alleviates oxidative stress in the liver with non- alcoholic steatohepatitis in rats. BMC Pharmacol Toxicol 17: 16, 2016.

18. McGahon AJ, Martin SJ, Bissonnette RP, Mahboubi A, Shi Y, Mogil RJ, Nishioka WK and Green DR: The end of the (cell) line: Methods for the study of apoptosis in vitro. Methods Cell Biol 46: 153-185, 1995. 
19. Chen H, Takahashi S, Imamura M, Okutani E, Zhang ZG, Chayama $\mathrm{K}$ and Chen BA: Earthworm fibrinolytic enzyme: Anti-tumor activity on human hepatoma cells in vitro and in vivo. Chin Med J (Engl) 120: 898-904, 2007.

20. Ribble D, Goldstein NB, Norris DA and Shellman YG: A simple technique for quantifying apoptosis in 96-well plates. BMC Biotechnol 5: 12, 2005 .

21. Lambert KE, Huang H, Mythreye K and Blobe GC: The type III transforming growth factor-beta receptor inhibits proliferation, migration, and adhesion in human myeloma cells. Mol Biol Cell 22: 1463-1472, 2011.

22. Lv C, Huang Y, Liu ZX, Yu D and Bai ZM: Salidroside reduces renal cell carcinoma proliferation by inhibiting JAK2/STAT3 signaling. Cancer Biomark 17: 41-47, 2016.

23. Hu X, Lin S, Yu D, Qiu S, Zhang X and Mei R: A preliminary study: The anti-proliferation effect of salidroside on different human cancer cell lines. Cell Biol Toxicol 26: 499-507, 2010.

24. Sun C, Wang Z, Zheng Q and Zhang H: Salidroside inhibits migration and invasion of human fibrosarcoma HT1080 cells Phytomedicine 19: 355-363, 2012.

25. Zhang L, Yu H, Sun Y, Lin X, Chen B, Tan C, Cao G and Wang Z: Protective effects of salidroside on hydrogen peroxide-induced apoptosis in SH-SY5Y human neuroblastoma cells. Eur J Pharmacol 564: 18-25, 2007.

26. Liu Z, Li X, Simoneau AR, Jafari M and Zi X: Rhodiola rosea extracts and salidroside decrease the growth of bladder cancer cell lines via inhibition of the mTOR pathway and induction of autophagy. Mol Carcinog 51: 257-267, 2012.

27. Li X, Su J, Xia M, Li H, Xu Y, Ma C, Ma L, Kang J, Yu H, Zhang Z and Sun L: Caspase-mediated cleavage of Beclin1 inhibits autophagy and promotes apoptosis induced by S1 in human ovarian cancer SKOV3 cells. Apoptosis 21: 225-238, 2016.

28. Zhang C and Qiu X: Andrographolide radiosensitizes human ovarian cancer SKOV3 xenografts due to an enhanced apoptosis and autophagy. Tumour Biol 36: 8359-8365, 2015.

29. Zhan Y, Xiang F, Wu R, Xu J, Ni Z, Jiang J and Kang X: MiRNA-149 modulates chemosensitivity of ovarian cancer A2780 cells to paclitaxel by targeting MyD88. J Ovarian Res 8: $48,2015$.
30. Huang $\mathrm{C}, \mathrm{Gu} \mathrm{H}$, Zhang W, Herrmann JL and Wang $\mathrm{M}$ : Testosterone-down-regulated Akt pathway during cardiac ischemia/reperfusion: A mechanism involving BAD, Bcl-2 and FOXO3a. J Surg Res 164: e1-e11, 2010.

31. Paulsen M, Ussat S, Jakob M, Scherer G, Lepenies I, Schütze S, Kabelitz D and Adam-Klages S: Interaction with XIAP prevents full caspase-3/-7 activation in proliferating human $\mathrm{T}$ lymphocytes. Eur J Immunol 38: 1979-1987, 2008.

32. Xie Y, Tobin LA, Camps J, Wangsa D, Yang J, Rao M, Witasp E, Awad KS, Yoo N, Ried T and Kwong KF: MicroRNA-24 regulates XIAP to reduce the apoptosis threshold in cancer cells. Oncogene 32: 2442-2451, 2013.

33. Ikenberg K, Valtcheva N, Brandt S, Zhong Q, Wong CE, Noske A, Rechsteiner M, Rueschoff JH, Caduff R, Dellas A, et al: KPNA2 is overexpressed in human and mouse endometrial cancers and promotes cellular proliferation. J Pathol 234: 239-252, 2014.

34. Li YD, Hong YF, Yusufuaji Y, Tang BP, Zhou XH, Xu GJ, Li JX, Sun L, Zhang JH, Xin Q, et al: Altered expression of hyperpolarization-activated cyclic nucleotide-gated channels and microRNA-1 and -133 in patients with age-associated atrial fibrillation. Mol Med Rep 12: 3243-3248, 2015.

35. Gu J, Tang Y, Liu Y, Guo H, Wang Y, Cai L, Li Y and Wang B: Murine double minute 2 siRNA and wild-type p53 gene therapy enhances sensitivity of the SKOV3/DDP ovarian cancer cell line to cisplatin chemotherapy in vitro and in vivo. Cancer Lett 343: 200-209, 2014

36. Gherman C, Braicu OL, Zanoaga O, Jurj A, Pileczki V, Maralani M, Drigla F, Braicu C, Budisan L, Achimas-Cadariu P and Berindan-Neagoe I: Caffeic acid phenethyl ester activates pro-apoptotic and epithelial-mesenchymal transition-related genes in ovarian cancer cells A2780 and A2780cis. Mol Cell Biochem 413: 189-198, 2016. 